\title{
EMBEDDINGS OF COMPACT SASAKIAN MANIFOLDS
}

\author{
Liviu ORNEA ${ }^{1}$ AND Misha VERBITSKY ${ }^{2}$
}

\begin{abstract}
Let $M$ be a compact Sasakian manifold. We show that $M$ admits a CRembedding into a Sasakian manifold diffeomorphic to a sphere, and this embedding is compatible with the respective Reeb fields. We argue that a stronger embedding theorem cannot be obtained. We use an extension theorem for Kähler geometry: given a compact Kähler manifolds $X \subset Y$, and a Kähler form $\omega_{X}$ on $X$ which lies in a Kähler class $[\omega]$ of $Y$ restricted to $X, \omega_{X}$ can be extended to a Kähler form $\omega_{Y}$ on $Y$.
\end{abstract}

\section{Introduction}

Sasakian manifolds are in many ways odd-dimensional counterparts of Kähler manifolds. Quite a few theorems of Kähler geometry have their analogues in Sasakian geometry.

In Kähler geometry one has the Kodaira-Nakano embedding theorem stating that a compact Kähler manifold with integral Kähler class is projective. The embedding given by this theorem is holomorphic but, in general, non-isometric.

Does there exist a similar result in Sasakian geometry?

To answer the question, one needs to find a model space (analogous of the projective space). One also needs to find out what is an analogue of holomorphic maps in Sasakian geometry.

Sasakian manifolds are equipped with a contact CR-structure and a CRholomorphic action of the corresponding Reeb field. An analogue of a holomorphic map is obviously a CR-holomorphic immersion $X \hookrightarrow Y$. One would also require that the Reeb field action on $Y$ preserve $X$.

In Kähler geometry, the model space for the Kodaira-Nakano embedding theorem is $\mathbb{C} P^{n}$. However, its analogue in Sasakian geometry must have continuous moduli, as we shall now explain. The CR-embeddings behave, in this sense, much more like Kähler immersions than the holomorphic immersions.

Recall that a Sasakian manifold where the Reeb field acts with compact orbits is called quasi-regular.

Consider a CR-holomorphic immersion of CR-manifolds $X \hookrightarrow Y$, with $Y$ Sasakian, and $X$ preserved by the Reeb field. This Reeb action defines a Sasakian structure on

Received by the editors November 2, 2006.

2000 Mathematics Subject Classification. 53C55, 53C25.

${ }^{1}$ Partially supported by CEx grant no. 2-CEx 06-11-22/25.07.2006.

${ }^{2}$ EPSRC advanced fellow supported by EPSRC grant GR/R77773/01. 
$X$. Indeed, it is positive in the sense of [BGS]. This is analogous to the fact that a restriction of a Kähler metric to a complex subvariety is again a Kähler metric. It turns out that any such a map is compatible with a set of Sasakian structures, and some of these Sasakian structures are quasi-regular.

Claim 1.1. Let $W_{1}, W_{2}$ be compact Sasakian manifolds and suppose there exists a CR-embedding $W_{1} \hookrightarrow W_{2}$ which commutes with the flows of the respective Reeb fields $\xi_{1}, \xi_{2}$. Then, there exists a quasi-regular Sasakian structure on $W_{2}$ whose Reeb flow preserves $W_{1}$.

Proof: Let $G$ be the group of Sasakian automorphisms of $W_{2}$ which preserve $W_{1}$. By assumption, $G$ contains the flow of $\xi_{2}$ and hence it contains a whole torus in which one can deform the initial Reeb flow to a quasi-regular one (see [OV2] and also [K]).

Let $\xi$ be this quasi-regular Reeb field on $W_{2}$ (its flow preserves $W_{1}$ ). It is a positive CR-vector field on $W_{2}$ and its restriction to $W_{1}$ is still CR-holomorphic and positive. Hence, according to [BGS], $\xi$ is a Reeb field on $W_{1}$ too. Then the orbifolds $W_{1} / \xi$ and $W_{2} / \xi$ are Kähler ${ }^{1}$, and one has the Kähler (thus isometric) embedding $W_{1} / \xi \hookrightarrow W_{2} / \xi$.

Now, if such a model space $W_{2}$ exists, the Kähler orbifold it fibers on is determined by a choice of a quasi-regular Reeb field. Such a Reeb field corresponds to a homomorphism $S^{1} \hookrightarrow T$, where the torus $T$ is the group of isometries of $W_{2}$ obtained as the closure of the flow of $\xi_{2}$ (see Claim 1.1). But there only exists a countable number of Lie group homomorphisms of $S^{1}$ into a compact torus. The source Sasakian manifold, $W_{1}$ in the above notations, projects on an arbitrary Kähler orbifold. And one cannot expect to have an isometric embedding of an arbitrary Kähler orbifold into a model Kähler orbifold, or a countable number of such orbifolds. Obviously, the model space must have continuous (and even infinite-dimensional) moduli.

This shows that one cannot aim at a precise analogue of the Kodaira-Nakano theorem for Sasakian manifolds (and, in particular, Theorem 6.1 in [OV2] is not correct as stated). ${ }^{2}$

Here we present the best possible result in this direction:

Theorem 1.2. Any compact Sasakian manifold admits a CR-embedding into a Sasakian manifold $S$ which is diffeomorphic to a sphere, and such that the corresponding Kähler manifold $S \times \mathbb{R}^{>0}$ is biholomorphic to $\mathbb{C}^{n} \backslash 0$.

We prove Theorem 1.2 in Section 3.

\section{Background on Sasakian and Vaisman manifolds}

We present the minimum necessary facts about Sasakian and Vaisman manifolds. For details and examples, see [BG, BGS, DO, OV1, OV2, OV3, OV4, Va].

\footnotetext{
${ }^{1}$ The Kähler structure on $W_{i} / \xi$ is obtained as a restriction of the Levi form.

${ }^{2}$ The argument used in the proof of [OV2, Theorem 6.1] is essentially correct, but only a contact immersion is constructed.
} 
Definition 2.1. A Sasakian manifold is a Riemannian manifold $(W, g)$ such that the cone metric $t^{2} g+d t^{2}$ on $W \times \mathbb{R}^{>0}$ is Kähler with respect to a dilatation-invariant complex structure.

The conic Kähler metric has a global potential $\varphi_{W}=t^{2}$. Clearly, $W$, identified with the slice at $t=1$, is the level set $\varphi_{W}^{-1}(1)$. As such, $W$ has a subjacent strictlypseudoconvex structure, the Reeb field being $\xi=J \frac{d}{d t}$ (see e.g. [OV4]). It can be shown that the Reeb field is Killing, unitary and all the plane sections passing through it have curvature 1 :

$$
R(X, Y) \xi=g(\xi, Y) X-g(X, Y) \xi .
$$

A pair $(g, \xi)$, with $\xi$ Killing, unit and satisfying $(2.1)$ is called a Sasakian structure on $W$.

The foliation generated by $\xi$ has a transverse Kähler structure. If the orbits of $\xi$ are closed, the Sasakian structure is called quasi-regular. In this case, the Reeb field generates a locally free $S^{1}$-action such that the leaf space is an orbifold and the transverse Kähler structure projects to it. Hence, a quasi-regular Sasakian manifold is a principal $S^{1}$-bundle over a Kähler orbifold.

Remark 2.2. All quasi-regular Sasakian manifolds can be obtained as follows (see [OV4, Example 1.9]). Let $X$ be a projective orbifold with only quotient singularities and let $L$ be an ample Hermitian line bundle over $X$ with positive curvature. Then $\operatorname{Tot}\left(L^{*} \backslash\{0\}\right)$, the space of non-zero vectors in the dual of $L$, has a Kähler structure given by the potential $\varphi(v)=|v|^{2}$. If $W:=\varphi^{-1}(1)$ is smooth, it is a Sasakian manifold which is a principal $S^{1}$-bundle over $X$.

While there are examples of Sasakian structures which are not quasi-regular (even Sasaki-Einstein ones, see e.g. [GMSW]), it is known that a Sasakian structure on a compact manifold can always be deformed to a quasi-regular one (see [OV2]). The trick is to deform the Reeb field in the torus generated by its flow.

Sasakian geometry is closely related to Vaisman geometry and our proof exploits this link. We confine to the compact case for which the following definition works (see [OV1]):

Definition 2.3. A Vaisman manifold is a Hermitian manifold $(M, J, h)$ admitting a cover which is a cone $W \times \mathbb{R}^{>0}$ endowed with a conic Kähler metric globally conformal with the lift of $h$ and with respect to which the deck group acts by holomorphic homotheties.

The Kähler metric in the definition has a global potential given by $\psi(m, t)=t^{2}$ (see $[\mathrm{Ve}])$.

The Reeb field of $W$ and its complex conjugate together give rise to a 1-dimensional holomorphic foliation $\mathcal{F}$ on $M$ which defines a transversal Kähler structure on a 
Vaisman manifold. When this foliation has compact leaves, the Vaisman structure is called quasi-regular and the leaf-space $M / \mathcal{F}$ is a Kähler orbifold.

It is known that the product of a compact Sasakian manifold with $S^{1}$ has a natural Vaisman structure.

A typical example of Vaisman manifold is the diagonal Hopf manifold

$H^{n}\left(\lambda_{1}, \ldots, \lambda_{n}\right):=\mathbb{C}^{n} \backslash\{0\} / \mathbb{Z} \cong S^{2 n-1} \times S^{1}$, where $\mathbb{Z}$ is represented by the group generated by $\left(z_{i}\right) \mapsto\left(\lambda_{i} z_{i}\right)$, for some nonzero complex numbers $\lambda_{i}$ of module $<1$. Hence, the Vaisman structure is here associated to a deformation of the standard Sasakian structure of the sphere. The orbifold $H^{n}\left(\lambda_{1}, \ldots, \lambda_{n}\right) / \mathcal{F}$ is in this case the weighted projective space $\mathbb{C} P^{n}\left(\lambda_{1}, \ldots, \lambda_{n}\right)$.

The Hopf manifold plays in Vaisman geometry the role of the projective space from Kähler geometry:

Theorem 2.4. [OV3] Every compact Vaisman manifold admits a holomorphic embedding in a diagonal Hopf manifold.

Now, starting with a compact quasi-regular Sasakian manifold, we consider a compact quasi-regular Vaisman manifold $W \times S^{1}$. Clearly, leaf-spaces $W / \xi$ and $M / \mathcal{F}$ are naturally identified. In particular, the weighted projective space can be viewed as $H^{n}\left(\lambda_{1}, \ldots, \lambda_{n}\right) / \mathcal{F}$ or as basis of the Hopf fibration $S^{2 n+1} / \xi$. Moreover, it can be shown ([OV2, OV3] $)$ that the above holomorphic embedding descends to a holomorphic embedding of the orbifold $W / \xi$ into the weighted projective space $\mathbb{C} P^{n}\left(\lambda_{1}, \ldots, \lambda_{n}\right)$.

On the other hand, any Vaisman structure has a subjacent locally conformal Kähler structure, i.e. there exists an open cover such that the restriction of the Vaisman metric to each open set of the cover is conformal to some Kähler metric. Hence, as in conformal geometry, one is led to consider the weight bundle $L^{\prime} \rightarrow M$ which is the trivial line-bundle associated to the frame bundle by the representation $\operatorname{GL}(2 n, \mathbb{R}) \mapsto \mid$ $\left.\operatorname{det}(A)\right|^{\frac{1}{2 n}}$, where $n=\operatorname{dim}_{\mathbb{C}} M$. We proved in [OV2] that for any compact quasiregular Vaisman manifold, the push-forward to $M / \mathcal{F}$ of the complexification of $L^{\prime}$ is ample. Using Remark 2.2, this gives a way of constructing quasi-regular compact Sasakian manifolds out of quasi-regular compact Vaisman ones.

\section{The embedding theorem}

Let $W$ be a compact Sasakian manifold, $M=W \times S^{1}$ the associated Vaisman manifold, and $\Psi: M \hookrightarrow H^{n}\left(\lambda_{1}, \ldots, \lambda_{n}\right)$ the holomorphic embedding into the diagonal Hopf manifold described in Theorem 2.4.

According to [OV4, Example 1.9], the Kähler cone $\widetilde{M}=W \times \mathbb{R}^{>0}$ of $M$ is equipped with a Kähler potential $\varphi_{W}$ such that $W:=W \times\{1\}=\varphi_{W}^{-1}(1)$. Then $\Psi$ naturally lifts to a holomorphic embedding $\widetilde{M} \hookrightarrow \widetilde{H^{n}}\left(\lambda_{1}, \ldots, \lambda_{n}\right)=\mathbb{C}^{n} \backslash\{0\}$.

Now assume that the potential $\varphi_{W}$ is the restriction of a potential $\varphi_{H}$ on $\mathbb{C}^{n} \backslash\{0\}$. Then we have a CR embedding $W \hookrightarrow \varphi_{H}^{-1}(\lambda)$. The following lemma implies that this space is diffeomorphic to a sphere. 
Lemma 3.1. Let $\varphi$ be a positive Kähler potential on $V=\mathbb{C}^{n} \backslash\{0\}$, satisfying $X_{A}(\varphi)=2 \varphi$ for a vector field $X_{A}$ on $V,\left.X_{A}\right|_{v}=A(v)$, where $A$ is a linear operator on $V$. Then the level sets $\varphi^{-1}(\lambda)$ are diffeomorphic to a sphere, for all positive $\lambda \in \mathbb{R}$.

Proof. Since $X_{A}(\varphi)=2 \varphi$, $\exp (A)$ acts on $V$ as a homothety, with respect to the Kähler metric defined by $\sqrt{-1} \partial \bar{\partial} \varphi$. Therefore, $\exp (-A)$ is a contraction.

Consider the 1-dimensional foliation $\mathcal{X}_{A}$ on $V$ generated by the vector field $X_{A}$. Let $r(t, x):=\exp (t A) x, x \in V, t \in \mathbb{R}$. Denote by $S_{x}$ the image of the map $r(\cdot, x): \mathbb{R} \longrightarrow V$. Clearly, the sets $S_{x}$ are leaves of $\mathcal{X}_{A}$. The leaf space $V / \mathcal{X}_{A}$ is naturally isomorphic to $S^{2 n-1}$ (this isomorphism is apparent, because $\exp (-A)$ is a contraction).

On $S_{x}$, the function $\varphi$ becomes exponential after a coordinate change. Therefore, $S_{x}$ intersects with $\varphi^{-1}(\lambda)$ precisely once. This defines a diffeomorphism

$$
\varphi^{-1}(\lambda) \longrightarrow V / \mathcal{X}_{A}=S^{2 n-1} \text {. }
$$

We thus have a CR embedding $W \hookrightarrow \varphi_{H}^{-1}(\lambda)$ in a Sasakian manifold diffeomorphic to a sphere.

Remark 3.2. This diffeomorphism is not a CR-equivalence. Indeed, CR-isomorphic pseudoconvex hypersurfaces in $\mathbb{C}^{n}$ bound domains which are biholomorphically equivalent (see e.g. [OV4]). On the other hand, the sphere $\varphi_{H}^{-1}(\lambda)$ is built from a homogeneous potential which can be chosen arbitrarily. In [OV4] it is shown that starting from an arbitrary strictly pseudoconvex, $S^{1}$-symmetric hypersurface $V \subset \mathbb{C}^{n}$, we can find a Kähler potential for which $V$ is a level set. Of course, a domain which is bound by such a hypersurface is not necessarily biholomorphic to a Euclidean ball.

From Lemma 3.1, we obtain that the embedding problem for Sasakian manifolds is reduced to an extension problem for plurisubharmonic functions which we now prove.

Theorem 3.3. Let $\Psi: W \times S^{1} \hookrightarrow H^{n}\left(\lambda_{1}, \ldots, \lambda_{n}\right)$ be the holomorphic embedding of Vaisman manifolds described above. Then there exists a Kähler potential on $\mathbb{C}^{n} \backslash\{0\}$ extending the potential $\varphi: W \times \mathbb{R}^{>0} \rightarrow \mathbb{R}$ and the restriction of $\Psi$ to the respective level sets is a CR-embedding.

Proof. Replace the Reeb flow on $W$ by a quasi-regular one as in Claim 1.1. Consider the holomorphic embedding $\Psi: W \times S^{1} \hookrightarrow H^{n}\left(\lambda_{1}, \ldots, \lambda_{n}\right)$ of quasi-regular Vaisman manifolds constructed as in [OV3]. Let $\widetilde{M} \rightarrow M$ be a cone covering of $M=W \times S^{1}$. We assume that $\widetilde{M}$ is endowed with a global Kähler potential given by the square length of the Lee field. As in [OV4], let $L \rightarrow X$ be an ample $\mathbb{C}^{*}$ Hermitian bundle over a projective orbifold $X$ such that $\widetilde{M}=\operatorname{Tot}\left(L^{*} \backslash\{0\}\right)$.

From the definitions it is apparent that the homogeneous weight 2 Kähler potentials on $\widetilde{M}$ are in one-to-one correspondence with the positive Hermitian structures on $L$.

As $X$ is projective, let $f: X \rightarrow \mathbb{C} P^{n}\left(\lambda_{1}, \ldots, \lambda_{n}\right)$ be a holomorphic embedding (in the category of orbifolds, see $[\mathrm{B}])$. Of course, $f$ is the projection of the holomorphic embedding $\Psi$. 
The bundle $L$ is the push-forward of the complexification of the weight bundle of the Vaisman manifold $W \times S^{1}$ (see [OV2]). As such, one sees that $L$ is the pull-back of the tautological bundle of the weighted projective space: $L=f^{*} O\left(\lambda_{1}, \ldots, \lambda_{n}\right)$. Hence, to finish the proof, it will be enough to show that the Hermitian metric of $L \rightarrow X$ is the restriction of a Hermitian metric in $O\left(\lambda_{1}, \ldots, \lambda_{n}\right) \rightarrow \mathbb{C} P^{n}\left(\lambda_{1}, \ldots, \lambda_{n}\right)$. But this will follow if we show that the metric (equivalently, the Kähler form) of $X$ is the restriction of a metric on $\mathbb{C} P^{n}\left(\lambda_{1}, \ldots, \lambda_{n}\right)$ preserving the given cohomology class, which is proven in Section 4.

\section{Kähler metrics extended from a submanifold}

4.1. Kähler metrics and plurisubharmonic functions. The sketch of the proof of the following theorem was communicated to us by J.-P. Demailly in an e-mail.

Theorem 4.1. Let $(M, \omega)$ be a compact Kähler manifold, and $Z \subset M$ a closed complex submanifold. Denote by $[\omega] \in H^{2}(M)$ the Kähler class of $M$. Consider a Kähler form $\omega_{0}$ on $Z$ such that its Kähler class coincides with the restriction $\left.[\omega]\right|_{Z}$. Then there exists a Kähler form $\omega^{\prime}$ on $M$ in the same Kähler class as $\omega$, such that $\left.\omega^{\prime}\right|_{z}=\omega_{0}$.

Proof. The proof is based on two theorems, which are found in [D]. The first one is existence of regularized maximum of two plurisubharmonic functions.

Proposition 4.2. Let $\varphi$ and $\psi$ be two smooth plurisubharmonic functions on a complex manifold $M$. Then for any $\delta>0$ there exists a smooth plurisubharmonic function $\max _{\delta}(\varphi, \psi)$ which is equal to $\max (\varphi, \psi)$ unless $|\varphi-\psi|<\delta$. Moreover, $\max _{\delta}(\varphi, \psi)$ is strictly plurisubharmonic if $\varphi$ and $\psi$ are strictly plurisubharmonic.

Proof. Let $\lambda: \mathbb{R}^{2} \longrightarrow \mathbb{R}$ be a convex function which is non-decreasing in each variable. Then $\lambda(\varphi, \psi)$ is also plurisubharmonic. Taking a smooth convex approximation $\lambda_{\delta}$ of $\lambda(x, y)=\max (x, y)$, we obtain $\max _{\delta}(\varphi, \psi):=\lambda_{\delta}(\varphi, \psi)$.

The second one is existence of a plurisubharmonic function with a logarithmic pole in any given complex variety.

Proposition 4.3. Let $(M, \omega)$ be a compact Kähler manifold and $Z \subset M$ a complex analytic subvariety. Then there exists a function $\varphi$ on $M$ which is smooth outside $Z$, satisfies

$$
\sqrt{-1} \partial \bar{\partial} \varphi>-C \omega
$$

for some positive constant $C$, and has logarithmic poles along $Z$; that is, locally along $Z$ we have

$$
\varphi(z) \sim \log \sum\left|g_{k}(z)\right|+O(1)
$$

where $g_{k}$ is a local system of generators of the ideal sheaf of $Z$ in $\mathcal{O}_{M}$.

Proof. See Lemma 2.1 of $[\mathrm{DP}]$. 
Using these two results, we can easily prove Theorem 4.1. Write the Kähler form $\omega_{0}$ as

$$
\omega_{0}=\left.\omega\right|_{z}+\sqrt{-1} \partial \bar{\partial} u
$$

where $u$ is a smooth function on $Z$. Extending $u$ to $M$ arbitrarily and adding the distance term $C_{1} d(z, Z)^{2}$, we may assume that $u$ is defined over $M$ and that (1$\varepsilon) \omega+i \partial \bar{\partial} u$ is strictly positive near $Z$, for some positive $\varepsilon<\frac{1}{2}$. Consider a function $\varphi$ with logarithmic poles in $Z$ constructed in Proposition 4.3. Near $Z$ the function $\varphi$ is very small, hence $\psi:=\max _{\delta}\left(\frac{\varepsilon}{C} \varphi+A, u\right)$ is equal to $u$ in some open neighbourhood of $Z$, for any constant $A \in \mathbb{R}$. Therefore, $\omega^{\prime}:=\omega+\sqrt{-1} \partial \bar{\partial} \psi$ satisfies $\left.\omega^{\prime}\right|_{Z}=\omega_{0}$. Let $U$ be a neighbourhood of $Z$ where $\sqrt{-1} \partial \bar{\partial} u+(1-\varepsilon) \omega$ is positive. The same argument as used to prove Proposition 4.2 also gives an estimate of $\left.\partial \bar{\partial} \psi\right|_{U}$ :

$$
\left.\sqrt{-1} \partial \bar{\partial} \psi\right|_{U} \geqslant-(1-\varepsilon) \omega
$$

(we use $\sqrt{-1} \frac{\varepsilon}{C} \partial \bar{\partial} \varphi \geqslant-\varepsilon \omega$, which comes from (4.1), and $\left.\sqrt{-1} \partial \bar{\partial} u\right|_{U} \geqslant-(1-\varepsilon) \omega$, which is essentially a definition of $U$; also, $\varepsilon<1-\varepsilon$, because we chose $\varepsilon<\frac{1}{2}$ ). Choosing $A$ sufficiently big, we may assume that $\psi=\varphi+A$ outside of $U$. Then, outside of $U, \omega^{\prime}$ is positive, because

$$
\left.\omega^{\prime}\right|_{M \backslash U}=\sqrt{-1} \partial \bar{\partial} \psi+\omega=\sqrt{-1} \frac{\varepsilon}{C} \partial \bar{\partial} \varphi+\omega>(1-\varepsilon) \omega
$$

which is positive. In $U$ the form $\omega^{\prime}$ is positive, as follows from (4.2):

$$
\omega+\sqrt{-1} \partial \bar{\partial} \psi \geqslant \omega-(1-\varepsilon) \omega=\varepsilon \omega .
$$

We proved Theorem 4.1.

4.2. Kähler forms on orbifolds. In order to prove Theorem 1.2, as indicated in Section 3, we need to apply the Kähler extension result (Theorem 4.1) to weighted projective spaces, which are orbifolds. The proof of Theorem 4.1 is valid in the orbifold situation, as we shall explain now.

A complex orbifold is a topological space covered by open sets of form $B / G$, where $B$ is an open ball in $\mathbb{C}^{n}$, and $G$ a finite group holomorphically acting on $B$ (different, generally speaking, for different elements of the covering), with the gluing map holomorphic and equivariant. It is Kähler if these open balls are equipped with a compatible Kähler metric, and the groups $G$ acts by isometries.

Results about differential forms and the Hodge theory are carried over to orbifolds word-by-word. In particular, the $\partial \bar{\partial}$-lemma is valid: given an exact $(p, q)$-form $\eta$ on a compact Kähler orbifold, we may always find $\eta_{1}$ such that $\eta=\partial \bar{\partial} \eta_{1}$.

The proof of Theorem 4.1 for orbifolds is done in the same way. We start with a smooth (in the sense of orbifolds) embedding $Z \subset M$, a Kähler form $\omega_{0}$ on $Z$, and a Kähler form $\omega$ on $M$ with $\left.\omega\right|_{Z}-\omega_{0}$ exact. Using $\partial \bar{\partial}$-lemma, we write

$$
\omega_{0}=\left.\omega\right|_{z}+\sqrt{-1} \partial \bar{\partial} u
$$

The rest of the argument is local, and is repeated verbatim.

Acknowledgements: We are grateful to J.-P. Demailly for invaluable help, Charles P. Boyer for insightful comments and to the referee for useful remarks. 


\section{References}

[B] W.L. Baily, On the imbedding of V-manifolds in projective spaces, Amer. J. Math. 79 (1957), 403-430.

[BG] C.P. Boyer, K. Galicki, Sasakian geometry, Oxford mathematical monographs, Oxford Univ. Press, 2006, to appear.

[BGS] C.P. Boyer, K. Galicki, S. Simanca, Canonical Sasakian metrics, math.DG/0504325.

[D] J.-P. Demailly, Estimations $L^{2}$ pour l'opérateur $\bar{\partial}$ d'un fibré vectoriel holomorphe semi-positif au-dessus d'une variété kählérienne complète, Ann. Sci. Ecole Norm. Sup. 4e Sér. 15 (1982) $457-511$.

[DP] J.P. Demailly, M. Paun, Numerical characterization of the Kähler cone of a compact Kähler manifold, Ann. of Math. 159 (2004), 1247-1274 [math.AG/0105176].

[DO] S. Dragomir and L. Ornea, Locally conformal Kähler geometry, Progress in Math. 155, Birkhäuser, Boston, Basel, 1998.

[GMSW] J. P. Gauntlett, D. Martelli, J. Sparks and D. Waldram, Sasaki-Einstein metrics on $S^{2} \times$ $S^{3}$, Adv. Theor. Math. Phys. 8, 711 (2004) [hep-th/0403002].

[K] Y. Kamishima, Standard pseudo-Hermitian structure on manifolds and Seifert fibrations, Ann. Global Anal. Geom., 12 (1994), 261-289.

[OV1] L. Ornea and M. Verbitsky, Structure theorem for compact Vaisman manifolds, Math. Res. Lett. 10 (2003), 799-805 [math.DG/0305259].

[OV2] L. Ornea and M. Verbitsky, An immersion theorem for compact Vaisman manifolds, Math. Ann. 332 (2005), no. 1, 121-143 [math.AG/0306077].

[OV3] L. Ornea and M. Verbitsky, Locally conformal manifolds with potential, math.AG/0407231.

[OV4] L. Ornea and M. Verbitsky, Sasakian structures on CR-manifolds, math.DG/0606136, to appear in Geometriae Dedicata.

[Va] Vaisman, I. Generalized Hopf manifolds, Geom. Dedicata 13 (1982), no. 3, 231-255.

[Ve] M. Verbitsky, Theorems on the vanishing of cohomology for locally conformally hyper-Kähler manifolds (Russian). Tr. Mat. Inst. Steklova 246 (2004), Algebr. Geom. Metody, Svyazi i Prilozh., 64-91; translation in Proc. Steklov Inst. Math. 2004, 246, 54-78 [math.DG/0302219].

University of Bucharest, Faculty of Mathematics,, 14 Academiei Str., 70109 Bucharest, ROMANIA.

E-mail address: lornea@gta.math.unibuc.ro, Liviu.Ornea@imar.ro

University of Glasgow, Department of Mathematics, 15 University Gardens, Glasgow, Scotland. Institute of Theoretical and Experimental Physics, B. Cheremushkinskaya, 25, Moscow, 117259, Russia

E-mail address: verbit@maths.gla.ac.uk, verbit@mccme.ru 